The correlations of optimal AV delays by non-invasive (Finometer) systolic blood pressure (SBP) vs invasive measures were as follows; aortic SBP, $\mathrm{r}^{2}=0.96, \mathrm{p}<0.01$; aortic flow velocity, $\mathrm{r}^{2}=0.81$, $\mathrm{p}<0.01 ; \mathrm{LV} \mathrm{dP} / \mathrm{dtmax}, \mathrm{r}^{2}=0.68, \mathrm{p}<0.01$.

Conclusions During acute biventricular pacing, at a fixed heart rate, changing the AV delay affects the cardiac mechanoenergetics. When an AV delay improves external cardiac work, compared to LBBB or a physiologically too short AV delay (eg, AV $40 \mathrm{~ms}$ ), it also increases the myocardial oxygen consumption. However, only $1 \%$ more energy is consumed per $1.6 \%$ more external work (pressure $\times$ flow) done; as a result cardiac efficiency improves. Haemodynamic optimisation of AV delay can be achieved with high precision using non invasive beat-to-beat pressure measurements. This should enable routine haemodynamic optimisation (easily automated) of CRT devices in clinical practice.

\section{ELECTROMECHANICAL INTERACTION IN PATIENTS UNDERGOING CARDIAC RESYNCHRONISATION THERAPY: COMPARISON OF INTRACARDIAC ACTIVATION MAPS AND EARLY SEPTAL CONTRACTION IN LEFT BUNDLE BRANCH BLOCK}

doi:10.1136/heartjnl-2011-300198.89

${ }^{1} \mathrm{~S}$ G Duckett, ${ }^{2} \mathrm{O}$ Camara, ${ }^{1} \mathrm{M}$ Ginks, ${ }^{3} \mathrm{~J}$ Bostock, ${ }^{1} \mathrm{P}$ Chinchapatnam, ${ }^{1} \mathrm{M}$ Sermesant, ${ }^{2} \mathrm{~A}$ Pashaei, ${ }^{3} \mathrm{~J} S \mathrm{~S}$ Gill, ${ }^{3} \mathrm{G}$ Carr-White, ${ }^{2} \mathrm{~A} F$ Frangi, ${ }^{1} \mathrm{R} S$ Razavi, ${ }^{2} \mathrm{~B} H$ Bijnens, ${ }^{3} \mathrm{C}$ A Rinaldi. ${ }^{1}$ Kings College London, London, UK; ${ }^{2}$ UPF, Barcelona, Spain; ${ }^{3} G u y$ 's and St Thomas' Hospital, London, UK

Introduction Early inward motion and thickening/thinning of the ventricular septum associated with left bundle branch block (LBBB) is known as the septal flash (SF). Correction of SF corresponds with CRT response. We hypothesise that electromechanical interactions induced by SF are associated with functional changes in conductivity and a "U-shaped" activation pattern. Characterising the spatio-temporal relationship between electrical and mechanical events may explain why patients with a SF respond to CRT. Methods 13 patients (63 \pm 10 years, 10 men) with severe heart failure (EF 22.8 $\pm 5.8 \%$ ) undergoing CRT underwent echocardiography and non-contact mapping (NCM) pre-implant. Presence and extent of a SF was defined visually and with $\mathrm{M}$-mode and fused with NCM bull's eye plots of endocardial activation patterns. LV $\mathrm{dP} / \mathrm{dtmax}$ was measured during different pacing modes.

Results Five patients had a large SF, four small SF and four no SF. Patients with large SF had areas of conduction block in noninfarcted regions whereas those with small or no SF did not (Abstract 89 figure 1). Patients with large SF had greater acute response to left ventricular (LV) and biventricular (BIV) pacing vs those with small/no SF (\% increase $\mathrm{dP} / \mathrm{dt} 28 \pm 14 \%$ vs $11 \pm 19 \%$ for LV pacing and $42 \pm 28 \%$ vs $22 \pm 21 \%$ for BIV pacing). The lines of conduction block disappeared after LV and BIV pacing, while remaining present with RV pacing (Abstract 89 figure 2). Abstract 89 figure 1 Patient with a large SF. Unipolar isochronal map with NCM electrograms showing fragmented signals (development of split potentials) indicating a reduction of conduction and inability to cross throughout the inferior region. The NCM mapping electrograms show the criteria used by Auricchio et al to define block, with the emergence of R-wave, smallest and earliest at the superior part of the block (where area of block begins) with largest negative peak. Bold white arrows on the electrogram indicate how the electrical activation spreads superiorly in a U-shape pattern leading to the development of split potentials. Abstract 89 figure 2 Activation maps of patient with a large SF. Row A, baseline with area of block and late anterior breakthrough. Row B, RV pacing showing the area of anterior block remains. Row C, BIV pacing. Functional conduction block has disappeared.

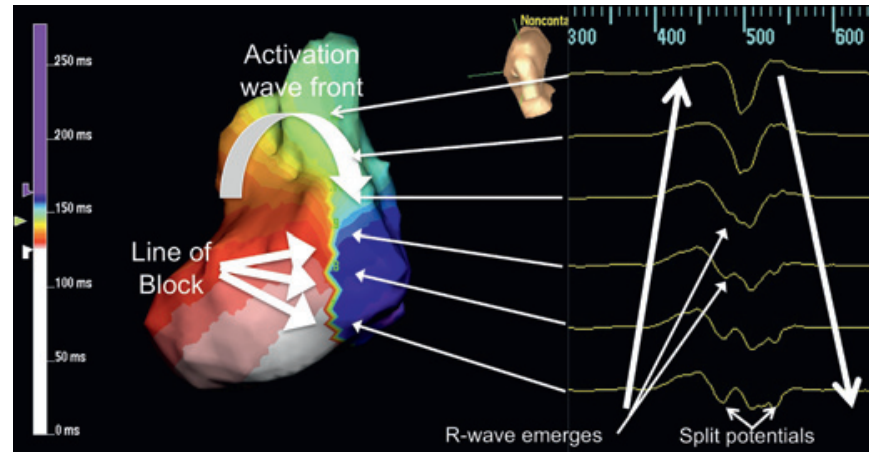

Abstract 89 Figure 1

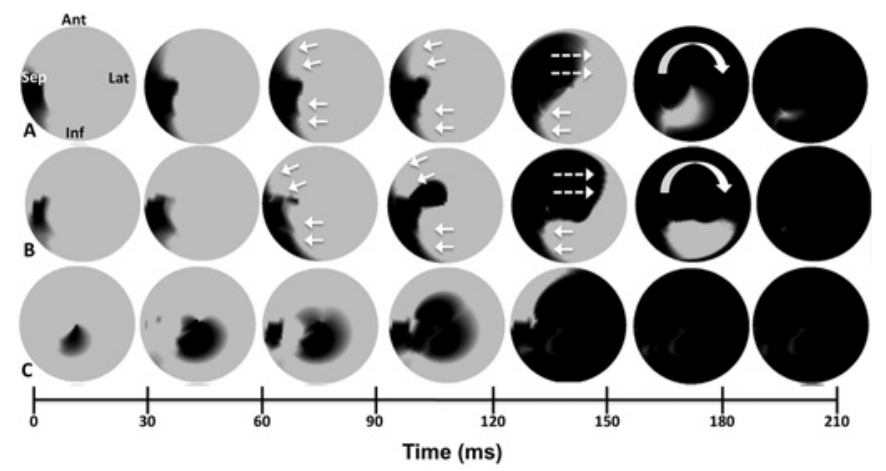

Abstract 89 Figure 2

Conclusion A strong interaction exists between electrical activation and mechanical deformation of the septum. Correction of both mechanical synchrony and the functional conduction block by CRT may explain the large positive response in patients with a SF.

\section{INVASIVE ACUTE HAEMODYNAMIC RESPONSE TO GUIDE LV LEAD IMPLANTATION PREDICTS CHRONIC REMODELLING IN PATIENTS UNDERGOING CARDIAC RESYNCHRONISATION THERAPY}

doi:10.1136/heartjnl-2011-300198.90

${ }^{1} \mathrm{~S}$ G Duckett, ${ }^{1} \mathrm{M}$ Ginks, ${ }^{1} \mathrm{~A}$ Shetty, ${ }^{2} \mathrm{~J}$ Bostock, ${ }^{2} \mathrm{~J} S \mathrm{~S}$ ill, ${ }^{2} \mathrm{~S}$ G Hamid, ${ }^{2} \mathrm{~S}$ Kapetanakis, ${ }^{2} \mathrm{E}$ Cunliffe, ${ }^{1} \mathrm{R} S$ Razavi, ${ }^{2} \mathrm{G}$ Carr-White, ${ }^{2} \mathrm{C}$ A Rinaldi. ${ }^{1}$ Kings College London, London, UK; ${ }^{2}$ Guy's and St Thomas' Hospital, London, UK

Introduction Cardiac resynchronisation therapy (CRT) reduces mortality and morbidity in heart failure patients, however up to 30\% of patients do not derive symptomatic benefit. Higher proportions do not remodel. Multi-centre trials have shown echocardiographic techniques are poor at improving response rates. We hypothesised that the degree of acute haemodynamic response (AHR) at implant can predict which patients remodel. We evaluated the relationship between AHR and reverse remodelling (RR) in CRT. Methods 33 patients undergoing CRT (21 dilated \& 12 ischaemic cardiomyopathy) were studied. Left ventricular (LV) volumes were assessed pre and post CRT. AHR (LV-dP/dtmax) was assessed at implant using a pressure wire in the LV cavity. The LV lead was placed in potential target veins and the largest percentage rise in $\mathrm{LV}-\mathrm{dP} / \mathrm{dtmax}$ from baseline (AAI or RV pacing with atrial fibrillation) to DDDLV was used to determine optimal LV lead position. RR was defined as reduction in LV end systolic volume (ESV) $\geq 15 \%$ at 6 months.

Results LV-dP/dtmax increased significantly from baseline $(801 \pm 194 \mathrm{~mm} \mathrm{Hg} / \mathrm{s}$ to $924 \pm 203 \mathrm{~mm} \mathrm{Hg} / \mathrm{s} \quad(\mathrm{p}<0.001))$ with DDDLV pacing for the optimal LV lead position. There was a significant difference in the percentage rise in LV-dP/dtmax between the best and worst LV lead position (Abstract 90 figure 1). LVESV 